This paper reports the analysis of patterns and mechanisms that form the characteristic surfaces of the Young modulus, the angular distributions of Poisson coefficients, and the pointing surfaces of auxeticity of single crystals in cubic, hexagonal, tetragonal, and rhombic crystal system. The crystals have been detected that can reach the limit negative values predicted by the classical elasticity theory for isotropic environments. It was found that near the points of phase transition or melting temperatures, the pointing surfaces of auxeticity rapidly increase, thereby turning the crystals into absolute auxetics. It is shozen that an array of negative Poisson coefficient values forms an image of the pointing surfaces of auxeticity. It is established that a reduction in the symmetry of crystals increases the number of crystallographic directions along which crystals gradually turn from "partial" to "mixed" or even "absolute" auxetics.

An analysis of the anisotropy of elastic properties, characteristic surfaces of the Young modulus, and the pointing surfaces of auxeticity has revealed that most single crystals of the highest and middle category barely reach the minimum limit values of Poisson coefficients. Therefore, in order to obtain more reliable auxetic materials with high impact-energy and seismic-resistant characteristics, it is necessary to investigate the anisotropy of elastic properties of low-category single crystals. The characteristic surfaces of the Young modulus have been constructed.

The volumetric images of the angular distributions of Poisson coefficients of the examined single crystals have been built, which make it possible to determine the absolute values and crystallographic orientation of the maximum and minimum values of Poisson coefficients. The pointing surfaces of the auxeticity of the studied single crystals have been constructed

Keywords: axial, non-axial auxeticity, Poisson coefficient, elasticity modules, pointing auxeticity surfaces
UDC 539.22: 539.32: 669.539.67

DOI: $10.15587 / 1729-4061.2020 .215167$

\section{DISCOVERING THE MECHANISMS THAT FORM THE AUXETIC PROPERTIES OF SINGLE CRYSTALS IN A MONOCLINIC CRYSTAL SYSTEM}

M. Raransk y

Doctor of Physical and Mathematical Sciences,

Professor*

E-mail: m.raransky@chnu.edu.ua

A. O l i i n y c h - L y s i u k $\mathrm{PhD}$, Associate Professor*

E-mail: a_oliynich@ukr.net

R. T a s h c h u k

Postgraduate Student*

E-mail: romatastshcuk@gmail.com

M. Un huria n *

E-mail: unhurian.mykhailo@chnu.edu.ua *Department of Information Technology and Computer Physics

Yuriy Fedkovych Chernivtsi National University Kotsiubynskoho str., 2, Chernivtsi, Ukraine, 58012
Received date 15.09.2020

Accepted date 21.10.2020

Published date 31.10.2020
Copyright (c) 2020, M. Raransky, A. Oliinych-Lysiuk, R. Tashchuk, M. Unhurian

This is an open access article under the CC BY license (http://creativecommons.org/licenses/by/4.0)
1. Introduction

The phenomenon of auxeticity is the occurrence, under certain conditions in the anisotropic environment, of the directions or regions of abnormal deformations with the negative Poisson coefficient values $\mu(\varphi, \theta, \psi)<0$. Auxetic materials, given their unique deformation-durable properties, are widely used for the manufacture of shock-absorbing and seismically resistant structures, protective equipment and filters, as well as various instruments and devices for functional acousto-optoelectronics. The physical nature of the phenomenon and the mechanisms that give rise to the auxetic properties of crystals are almost not studied and require detailed research.

At small deformations, the elastic properties of homogeneous isotropic environments are described by the Young modulus $E$ modules, the shear modulus $F$, full compression $B$, and the Poisson coefficients $\mu$, which are the fundamental constants of structural materials. It follows from the requirements for the positivity of the specific potential energy of deformation that the values $E, B, G$ are positive while the Poisson coefficients $\mu$ should be in the range of $-1 \leq \mu \leq 0.5$. Thus, from a thermodynamic point of view, the existence of materials with a negative value $\mu<0$ is not ruled out. However, for most crystals, $\mu \leq 0.5$, and the negative Poisson coefficient values generally contradict the laws of classical elasticity theory. Therefore, investigating the abnormal deformations with negative Poisson coefficient values was not paid much attention to; there are almost no relevant studies.

At the end of the twentieth century, a large number of new materials with unique deformation properties were discovered, for which $\mu<0$. These materials, at the longitudinal elongation in certain crystallographic directions, expand in transverse sizes and are termed auxetics.

Most auxetics are anisotropic bodies whose elastic properties are described by tensor values. Note that the number 
of independent components of the elasticity modules' tensor $C_{i j k l}$ is the greater the lower the symmetry of the crystals. Consequently, for a proper analysis of the anisotropy of elastic properties and auxeticity of the examined single crystals, it is necessary to build the pointing or characteristic surface of the Young modulus $E_{\mathrm{i}}$ and the Poisson coefficients $\mu_{i j k l}$.

\section{Literature review and problem statement}

In the first stage of scientific research, the focus of most researchers was on finding new crystals - auxetics [1-3]. The negative Poisson coefficient values were found in crystals, at least in some crystallographic directions or at the fixed values of one or two Euler angles $\varphi, \theta, \psi[3-6]$.

The issue is that it is impossible to build a four-dimensional surface of the Poisson coefficients $\mu(\varphi, \theta, \psi)$ in a three-dimensional coordinate system. Therefore, no patterns in the formation of auxetic surfaces of crystals were investigated.

An overview of the basic auxetic properties of composite materials, crystalline bodies, and molecular crystals is given in work [1]. It is shown that the auxetic properties of materials are due to the "structural and mechanical features of their structure" and the physicochemical processes occurring in them. The largest number of auxetics was found among metals and intermetallic phases, some semiconductors and alkaline halide compounds mainly in a cubic crystal system. A generalized criterion for the "auxetic behavior" of single crystals in a cubic crystal system was proposed in [2]. It was found that the negative Poisson coefficient values are concentrated in the $<110>$ directions, that is, cubic crystals are the non-axial auxetics.

The deformation of auxetic crystals of different crystal systems is considered in work [3]. However, [3] was limited to analyzing the uni-axial deformation and the dependence of the elastic characteristics of crystals on one of the Euler angles only. The expressions for the Young modulus $E(\theta)$ and the Poisson coefficients $\mu(\theta)$ were derived. Along with the analysis of experimental values and the periodicity of function $E(\theta)$ and $\mu(\theta)$, the cited work does not examine the relationship between the anisotropy of elastic properties and the auxeticity of the studied single crystals. An attempt to establish a correlation between the single-parametric functions $E(\theta)$ and $\mu(\theta)$ is reported in [4]. For this purpose, the Young modulus of more than 350 cubic auxetic crystals in three experimental directions $E_{[100]}, E_{[110]}$, and $E_{[111]}$ was calculated. It is shown that "cubic auxetics have a high degree of anisotropy". For the "strongly anisotropic" crystals ( $\mathrm{Li}$, $\mathrm{Na}, \mathrm{K}, \mathrm{In}, \mathrm{Te}$ ), the Young modulus, depending on direction, can vary by $5-7$ times. The variability of the Young modulus depends significantly on the elastic anisotropy coefficient $\Delta=S_{11}-S_{12}-0.5 S_{44}$, where $S_{i j}$ is the components of an elastic pliability tensor. It was found that some cubic crystals (Ba, $\left.\mathrm{Sm}_{x} \mathrm{La}_{1-x} \mathrm{~S}, \mathrm{Sm}_{x} \mathrm{Y}_{1-x} \mathrm{~S}\right) \times \mathrm{S}$ ) are the absolute auxetics, that is, for them $\mu<0$ in almost all crystallographic directions.

Paper [5] defines the "anisotropic and isotropic" Poisson coefficients for some ionic and ionic-covalent crystals with a type $x$ lattice, and $\mathrm{ZnS}$. It was established that the extreme values of Poisson coefficients "do not depend on the type of crystal lattice" but are determined by the multiplier of elastic anisotropy. The authors calculated the temperature (for $\mathrm{LiF}$ and $\mathrm{NaCN}$ ) and baric (for $\mathrm{CuCl}$ ) dependences of the Poisson coefficients. Paper [5] proposes the introduction of a "criterion" of non-axial auxeticity. Note that this criterion was introduced much earlier in work [2].

Paper [6] reported a comprehensive experimental study into the anisotropy of elastic properties and the anharmonicity of atom fluctuations in the crystalline lattice of single crystals in the cubic, hexagonal, trigonal, orthorhombic, and monoclinic crystal systems. The ultrasonic and X-wave methods were used to determine the concentration and temperature dependences of ultrasonic wave propagation velocity in the $v(x, T)$ crystals, as well as the $a_{i}(x, T)$ lattice periods, in single crystals $\mathrm{NaCl}, \mathrm{KCl}, \mathrm{LiF}, \mathrm{CaF}_{2}, \mathrm{ZnTe}, \mathrm{CdTe}$, $\mathrm{HgTe}, \mathrm{CdSb}, \mathrm{ZnSb}, \mathrm{In}_{2} \mathrm{Se}_{3}, \mathrm{Bi}_{2} \mathrm{Te}_{3}, \mathrm{ZnAs}_{2}$.

The modulus of elasticity $C_{i j}(x, T)$, shear modules $G_{s}$ $(x, T)$, the modules of full compression $B_{i}(x, T)$, and other elastic characteristics of crystals, were calculated. For the first time, the characteristic surfaces of the Young modulus for single crystals of the higher, middle, and lower categories were built, intersecting which by the $(h k l)$ crystallographic planes makes it possible to obtain the numerical values $E_{<h k l>}$ in arbitrary directions. The combination of acoustic and X-wave diffraction methods allowed the authors to unambiguously determine the main parameters of crystal lattice dynamics, the characteristic Debye temperatures $\theta_{D}(x T)$, the Grunaisen parameters $\gamma_{i}(x, \mathrm{~T})$. It is shown that the anisotropy of elastic properties and the anharmonicity of atom fluctuations in a crystalline lattice can lead to abnormal deformities of crystals.

In the second stage of scientific research, there is a task of studying the mechanisms of abnormal deformation of single crystals, which leads to the occurrence of the negative values of Poisson coefficients. Most research in [1-5] is limited to the calculation of the Young modulus $E_{i}$, the shear module $G_{s}$, and the Poisson coefficients $\mu_{i j}$ only in certain crystallographic directions. More detailed information about the anisotropy of the elastic properties of single crystals can be obtained by building the characteristic surfaces of these values.

The formation of the characteristic surfaces of the Young modulus for single crystals in a cubic crystal system with different types of crystal lattices (BCC, FCC, $\mathrm{NaCl}, \mathrm{GsCl}$, $\mathrm{CaFe}, \mathrm{ZnS}$ ) and the character of chemical bond (metallic, ionic, ionic-covalent) were analyzed in detail in work [7]. It was found that the mechanisms that form the characteristic surfaces of the Young modulus are clearly associated with the values $C_{i j}$, which adequately reproduce the nature of the forces of interatomic interaction in different crystals. Therefore, the characteristic surfaces retain all elements of the symmetry of the studied crystals. It was established that the shape of the characteristic surfaces is determined by the parameter of elastic anisotropy $=C_{44} / G_{s}$, where $G_{s}=\left(C_{11}-C_{12}\right) / 2$ is a shear module that is proportional to the energy of the covalent bond and is determined by the disorientation of hybridized $\mathrm{sp}^{3}$-orbitals [8]. In terms of the shape of the characteristic surfaces of the Young modulus explored in [7], crystals can be conditionally divided into three types. At $G_{s}<C_{44}$, the elastic anisotropy factor $A>0$ and the characteristic surface of the Young modulus take the shape of a deformed cube with rounded vertices and edges and concave face surfaces, and the extreme values $E_{<111>}>E_{<110>}>E_{<100>}$. This surface shape is typical of metals with BCC and FCC lattices and semiconductors with lattices the type of diamond and $Z n S$. If $G_{s}=C_{44}$ then $A=1$ and the characteristic surface of the Young modulus degenerates from a sphere into an octahedron with rounded vertices and edges, and the extreme values of the Young modulus $E_{<100>}>E_{<110>}>E_{<111>}$. This shape is typical 
of single crystals with lattices such as $\mathrm{NaCl}, \mathrm{CsCl}, \mathrm{CaF}_{2}$, and chalcogenide semiconductor compounds $\mathrm{PbS}, \mathrm{PbSe}, \mathrm{PbTe}$, SnTe (except for Li haloids, for which $C_{44}>G s$ ). For mediumand lower-order single crystals, the number of independent components of the elasticity module tensor $C_{i j}$ increases, and the anisotropy of elastic properties grows rapidly [6]. There is also an increase in the number of elastic anisotropy parameters $A_{i}$ that are the linear combinations of $C_{i j}$. Note that the extreme values of the Young modulus $E_{<h k l}$ can be directed in other crystallographic directions. Regularities of formation of the characteristic surfaces of the Young modulus of middle- and lower-category single crystals are described in [9].

By analogy with the characteristic surfaces of the Young modulus, the concepts of pointing surfaces of the Poisson coefficients are introduced. Due to the presence of certain elements of symmetry in real single crystals, the function $\mu(\varphi, \theta, \psi)$ is periodic. Analysis of the functions $\mu(\varphi, \theta, \psi)$ shows that the negative Poisson coefficient values $\left(\mu_{\min }<0\right)$ are concentrated in certain crystallographic directions and form some surfaces in space. However, the angular distribution of the $\mu(\varphi, \theta, \psi)$ function can only be constructed at the fixed values for one of the Euler angles. Work [10] reports a study into the single-parametric change in the surfaces $\mu(\varphi, \theta, \psi)=0$ that divide regions with the negative $\left(\mu_{\min }<0\right)$ and positive $\left(\mu_{\max }>0\right)$ values of the Poisson coefficients. It was found that for cubic crystals, the "dividing surface" has a "rather complex topological structure" that "disintegrates into some set of closed surfaces." The concept of "delineation curves $\mu(\varphi=0, \theta, \psi)=0$ " was introduced, separating the regions of " auxetic (at $\mu<0)$ and non-auxetic (at $\mu>0)$ behavior» of crystals. For the tetragonal crystals, work [11] termed the "separating" surface $\mu(\varphi, \theta, \psi)=0$ an "auxeticity surface". Extreme values of the Poisson coefficients were determined based on a change in the surfaces of the equation $\mu(\varphi, \theta, \psi)=C$, where $0 \leq C \leq 1$ is a dimensionless parameter. Note that the analysis of the auxeticity surfaces of the studied crystals, conducted in works [10,11], was not effective. First, there is no information on the type of crystalline lattices, the spatial group of symmetry, the nature of the chemical bond, and the type of crystal auxeticity. Second, crystallographic directions are not specified, in which the extreme values of the Young modulus $\left(E_{\min }, E_{\max }\right)$ and the Poisson coefficients $\left(\mu_{\min }, \mu_{\max }\right)$ are concentrated. Third, it is not correct to compare the "variability of the Young modulus" with the "negative" values of the Poisson coefficient for crystals with unknown types of auxeticity. Even crystals belonging to the same crystal system can have different types of auxeticity (axial, non-axial, mixed) and, consequently, different directions of abnormal deformity [9].

Therefore, the authors of [11] came to a strange conclusion about "... no link between the variability of the Young modulus and the negative Poisson coefficient values for 7 constant tetragonal crystals was detected".

To analyze the patterns in the formation of the characteristic surfaces $\mu(\varphi, \theta, \psi)$, we have developed a program that makes it possible to calculate, based on certain ratios, the magnitude $\mu$, choose the negative values $\mu_{\min }<0$, and their respective directions, and build the surfaces of auxeticity. An array of negative Poisson coefficient values will form in space some surface, which we term the pointing auxeticity surface.

As shown in works $[12,13]$, for crystals of the cubic crystal system $(\mathrm{CdTe}, \mathrm{LiF}, \mathrm{Pd}) \mu_{\min }=-0.01$, the auxetic surface is formed in the form of 12 separate points in the $<110>$ crystallographic direction. For crystals of Au, Ag, $\mathrm{Cu}$, with the growth of the elastic anisotropy parameter $A$ and a decrease in $\mu_{\min }$ (from $] 0.03$ to -0.19 ), there is a gradual increase in the size of points and the formation of spots. Further increase in $A$ and a decrease in $\mu_{\min }$ for $\mathrm{Li}, \mathrm{K}, \mathrm{Na}$, $\mathrm{Rb}$ crystals lead to the formation of a surface in the form of spherical belts.

Consequently, the area of the pointing auxeticity surfaces $S$ increases in proportion to the growth of parameter $A$ and a decrease in $\mu_{\min }$. And only near the phase transition points $\left(\Theta_{D}=78 \mathrm{~K}\right.$ for $\left.\mathrm{Li}\right)$ or melting points $\left(T_{\text {melt }}=1143 \mathrm{~K}\right.$, for $\mathrm{LiF})$, the area of the pointing auxeticity surfaces $S$ increases and gradually approaches the area of the sphere. There is an unambiguous correlation between the values $\mathrm{A}, \mu_{\min }, S$. Extreme values of the Poisson coefficients for cubic crystal system's crystals were found for a $\mathrm{NiCrO}^{4}$ single crystal: $\mu_{\min }=-0.77 ; \mu_{\max }=1.72$.

For hexagonal crystal system's single crystals, an array of negative values $\mu_{\min }<0$ forms in space the images of the pointing auxeticity surfaces in the form of spherical segment belts [14]. The Poisson coefficient negative values are concentrated in 8 crystallographic equivalent directions, the type of [hk0], [0k0], $h=k=+/-1$ (Be, TiBe, $\mathrm{BeCu}, \mathrm{Zn}$, $\mathrm{C}_{7} \mathrm{H}_{14} \mathrm{MoS}_{2}-$ mixed auxeticity, the crystals are arranged in order of descending negative values $\mu_{\min }$. Extreme values of the Poisson coefficients are found for $\mathrm{MoS}_{2}$ crystals $\left(\mu_{\min }=-0.28\right)$ and $\mathrm{C}_{7} \mathrm{H}_{14}\left(\mu_{\max }=0.99\right)$. The area of pointing auxeticity surfaces $S$ increases in proportion to the reduction of negative values $\mu_{\min }$ and the parameter of anisotropy $A_{1}=C_{33} / C_{11}$. When the Be crystals are heated, there is an abnormal deformity and, consequently, a cascade of transitions: non-axial $\rightarrow$ axial $\rightarrow$ absolute auxetic. Consequently, the division of the hexagonal crystal system's crystals into axial and non-axial crystals is not correct. Patterns in the formation of the characteristic surfaces of the Young modulus for single crystals with CPH lattice are described in work [15].

The negative Poisson coefficient values of the single crystals in the tetragonal crystal system $\left[\mathrm{NH}_{4} \mathrm{H}_{2} \mathrm{PO}_{4}\left(\mu_{\text {min }}=\right.\right.$ $=-0.12), \mathrm{K} \mathrm{H}_{2} \mathrm{PO}_{4}\left(\mu_{\min }=-0.13\right) \mathrm{RbH}_{2} \mathrm{AsO}_{4}\left(\mu_{\min }=-0.37\right)$ and $\left.\mathrm{RbD}_{2} \mathrm{AsO}_{4}\left(\mu_{\min }=-0.41\right)\right]$ are concentrated in 4 crystallographic directions [ $h 00]$ and [0k0] $(h=k=+/-1)$. They form the pointing surface in the form of vertical bands, which, when $\mu_{\text {min }}$ is reduced, are gradually converted into spherical segments.

The directions of extreme values of the Young modulus and Poisson coefficients coincide [15]. The area of the pointing auxeticity surfaces $S$ increases in proportion to the growth of $E_{\max } / E_{\min }$, also accompanied by a decrease in $\mu_{\min }$ (from -0.1 to -0.4$)$. For $\mathrm{Hg}_{2} \mathrm{Cl}_{2}$ single crystals $\left(\mu_{\min }=-0.91\right), \mathrm{Hg}_{2} \mathrm{I}_{2}$ $\left(\mu_{\min }=-0.96\right)$, and $\mathrm{Hg}_{2} \mathrm{Br}_{2}\left(\mu_{\min }=1.02\right)$, the pointing auxeticity surface degenerates into a sphere. Consequently, negative values $\mu(\varphi, \theta, \psi)$ are observed in all crystallographic directions (almost absolute auxeticity). The area of the pointing surface $S$ increases in proportion to the increase in the ion radiuses of cations $r_{i}$ and the relative periods of crystalline lattices $c / a$. The extreme values of the Poisson coefficients for tetragonal crystals are as follows: $\mathrm{Hg}_{2} \mathrm{Br}_{2}, \mu_{\min }=-1.02 ; \mathrm{Hg}_{2} \mathrm{I}_{2}, \mu_{\max }=1.99$ [3, 16].

Based on the analysis of the anisotropy of elastic properties of single crystals from the higher and middle categories, the main mechanisms and patterns in the formation of pointing auxeticity surfaces are established. The directions of orientation of the negative values of Poisson coefficients $\mu_{\min }$ have been determined. It is shown that with the reduction of symmetry, the number of directions $\mu_{\min }$ increases and 
crystals gradually become mixed or even absolute auxetics at room temperature. For example, for the single crystal in a hexagonal crystal system $\mathrm{Zn}\left(\mu_{\min }=-0.07\right)$, the negative Poisson coefficient values are concentrated in 8 crystallographic directions [ $h k 0]$ and [h00], [0k0]; $h=k=+/-1$ (non-axial and axial auxeticity). For a single crystal in the rhombic crystal system $\mathrm{AgN}_{3}\left(\mu_{\min }=-0.5\right)$ - in 12 separate directions ( 4 from each set of directions $<110>$ and $<111>$ and two more in the $[0 k 0]$ and $[00 l]$ directions) [9].

Of course, a significant angular division $\mu$ leads to their overlap and formation of the auxeticity surfaces in the form of rather complex three-dimensional shapes. For each crystal system, crystals were found in the higher and middle categories, which not only reached the limit positive values $\mu_{\max }=0.5$, predicted by the theory of elasticity for isotropic materials, but also surpassed them.

At the same time, the minimum limit values of the Poisson coefficients $\left(\mu_{\min }=-1.00\right)$ are barely reached by only two crystals of $\mathrm{Hg}_{2} \mathrm{Br}_{2}\left(\mu_{\min }=-1.00\right.$, tetragonal crystal system) and $\mathrm{NaNO}_{3}\left(\mu_{\min }=-0.99\right.$, trigonal crystal system) [9].

The experimental values of the elasticity modules $C_{i j}$ and pliability $S_{i j}$ [16] were used to calculate the angular distributions of the Poisson coefficients $\mu(\varphi, \theta, \psi)$ and for the first time the characteristic surfaces of the Young modulus and the pointing auxeticity surfaces for single crystals in a monoclinic crystal system were built.

The anisotropy of the elastic properties of these crystals is analyzed. There is hope that due to the low symmetry and the high degree of anisotropy single crystals in monoclinic crystal systems will significantly surpass both the lower $\left(\mu_{\min }\right)$ and upper $\left(\mu_{\max }\right)$ boundaries of permissible values of the Poisson coefficients. The research is a continuation of a series of studies carried out at the Department of Solid State Physics (Yuriy Fedkovych Chernivtsi National University) aimed at investigating the mechanisms and patterns in the formation of auxetic properties, the nature of chemical bonds, and the dynamics of crystalline lattices of single crystals in the high, medium, and low categories.

\section{The aim and objectives of the study}

The aim of this work is to identify mechanisms that form the auxetic properties of single crystals in a monoclinic crystal system. This would make it possible to determine the features of abnormal deformation and the anisotropy of elasticity of low-category single crystals and identify single crystals with the extreme values of Poisson coefficients.

To accomplish the aim, the following tasks have been set:

- to build the characteristic surfaces of the Young modulus for single crystals of labradorite, calcium sulfate dihydrate, zirconium oxide, fluoroduodecane, cesium dihydrogen phosphate, and lanthanum niobate;

- to calculate the Poisson coefficients for these single crystals in all possible crystallographic directions, determine the directions at which $\mu_{\min }<0$, and to build the pointing auxeticity surfaces;

- to detect the effect of the elastic anisotropy of single crystals on the formation of the characteristic surfaces of Young modulus and pointing auxeticity surfaces;

- to establish the relationship between the degree of auxeticity $S_{a}$ and the extreme values of Young modulus and Poisson coefficients.

\section{Theoretical basis of the auxeticity of single crystals in a monoclinic crystal system}

The statistics of the distribution of crystals based on crystal systems and the classes of symmetry are quite uneven. Among 30,000 examined minerals and synthesized crystals, more than half ( $\sim$ than $53.5 \%$ ) are in a low category $[17,18]$. Of these, crystals of the monoclinic crystal system occupy the top place ( 26-27\%). Due to their high anisotropy, a large number of auxetics should be expected. However, there are almost no studies into the anisotropy of elastic properties of the monoclinic crystal system's crystals. This is due to the fact that such studies require perfect single crystals of large size. Only two works are known, in which the one-parameter dependences $E(\theta)$ and $\mu(\theta)$ for some single crystals were calculated [3] and the characteristic surface of the Young modulus for the $\mathrm{ZnAs}_{2}$ single crystal was constructed [6].

There are only three classes of symmetry in the monoclinic crystal system: $2, m$, and $2 / m$ (international symbols). They have the following elements of symmetry: either one second-order axis $L_{2}$, or one plane $P$, or compatible $P L_{2}$ and $C$, respectively, where $C$ is the center of symmetry. The periods of the crystalline lattice are $a \neq b \neq c$; the angles between the planes $\alpha=\gamma \neq 90^{\circ}$. As a result, there is ambiguity in the selection of the $X Y Z$ orthogonal coordinate system. If one places the $X$ and $Y$ axes in plane $P$, and the $Z$ axis - perpendicular to it, or along the second-order axis $L_{2}$ (in the presence of such), the pliability module matrix takes the form [19]:

$$
S_{i j}=\left(\begin{array}{cccccc}
S_{11} & S_{12} & S_{13} & 0 & S_{15} & 0 \\
S_{12} & S_{22} & S_{23} & 0 & S_{25} & 0 \\
S_{31} & S_{23} & S_{33} & 0 & S_{35} & 0 \\
0 & 0 & 0 & S_{44} & 0 & S_{46} \\
S_{15} & S_{21} & S_{35} & 0 & S_{55} & 0 \\
0 & 0 & 0 & S_{46} & 0 & S_{66}
\end{array}\right) .
$$

Consequently, the elastic properties of the monoclinic crystal system's crystals are described by thirteen independent components of the pliability modules $S_{i j}$. For the symmetry class $2 / m$ crystals (symmetry formula is $L_{2} P C$ ) with proper selection of the coordinate system, the number of independent components can be reduced to 12. The criterion for the thermodynamic stability of the crystalline lattices of single crystals is the positivity of the determinants of all matrices of successive ranks of the quadratic form (1).

To unequivocally determine the values and signs of pliability modules, it is necessary to measure at least 13-18 speeds of the propagation of longitudinal and transverse US waves along six crystallographic directions: [100], [010], [001], [110], [101], and [011]. The correlations between the speeds of US waves and the $S_{i j}$ modules are quite cumbersome and are not given here, they can be found in [20]. The methodology for calculating the modules of some semiconductor and molecular crystals in the monoclinic crystal system is given in $[6,21]$.

To analyze the effect of anisotropy of elastic deformations on the auxetic properties of single crystals, it is necessary to build the characteristic surfaces of the Young modulus and the pointing auxeticity surfaces. Given that for single crystals in the monoclinic crystal system $S_{14}=S_{16}=S_{24}=S_{26}=S_{34}=$ 
$=S_{36}=S_{45}=S_{46}=0$, the expression for the Young modulus shall be written in the form [17]:

$$
E(\vec{q})=1 /\left(\begin{array}{l}
q_{1}^{4} S_{11}+2 q_{1}^{2} q_{2}^{2} S_{12}+2 q_{1}^{2} q_{3}^{2} S_{13}+ \\
+2 q_{1}^{3} q_{3} S_{15}+q_{2}^{4} S_{22}+2 q_{2}^{2} q_{3}^{2} S_{23}+ \\
+2 q_{1} q_{2}^{2} q_{3} S_{25}+q_{3}^{4} S_{33}+ \\
+2 q_{1} q_{3}^{3} S_{35}+q_{2}^{2} q_{3}^{2} S_{44}+ \\
+2 q_{1} q_{2}^{2} q_{3} S_{46}+q_{1}^{2} q_{3}^{2} S_{55}+q_{1}^{2} q_{2}^{2} S_{66}
\end{array}\right),
$$

where $q_{i}$ is the single vectors; $S_{i j}$ is the module of elastic pliability.

The Poisson coefficient is equal to:

$$
\begin{gathered}
\mu_{i j}=-\left[\begin{array}{l}
\left(\alpha_{11}^{2} \alpha_{21}^{2}\right) S_{11}+\left(\alpha_{11}^{2} \alpha_{22}^{2}+\alpha_{12}^{2} \alpha_{21}^{2}\right) S_{12}+ \\
+\left(\alpha_{11}^{2} \alpha_{23}^{2}+\alpha_{13}^{2} \alpha_{21}^{2}\right) S_{13}+ \\
+\left(\alpha_{11}^{2} \alpha_{21} \alpha_{23}+\alpha_{21}^{2} \alpha_{11} \alpha_{13}\right) S_{15}+ \\
+\left(\alpha_{12}^{2} \alpha_{22}^{2}\right) S_{22}+\left(\alpha_{12}^{2} \alpha_{23}^{2}+\alpha_{22}^{2} \alpha_{13}^{2}\right) S_{23}+ \\
+\left(\alpha_{11} \alpha_{13} \alpha_{22}^{2}+\alpha_{21} \alpha_{23} \alpha_{12}^{2}\right) S_{25}+ \\
+\alpha_{13}^{2} \alpha_{23}^{2} S_{33}+\left(\alpha_{11} \alpha_{13} \alpha_{23}^{2}+\right. \\
\left.+\alpha_{21} \alpha_{23} \alpha_{13}^{2}\right) S_{35}+\alpha_{12} \alpha_{13} \alpha_{22} \alpha_{23} S_{44}+ \\
+\left(\alpha_{11} \alpha_{12} \alpha_{22} \alpha_{23}+\right. \\
\left.+\alpha_{21} \alpha_{22} \alpha_{12} \alpha_{13}\right) S_{46}+\alpha_{11} \alpha_{13} \alpha_{21} \alpha_{23} S_{55}+ \\
+\alpha_{11} \alpha_{12} \alpha_{21} \alpha_{22} S_{66}
\end{array}\right] / \\
/\left[\begin{array}{l}
\alpha_{21}^{4} S_{11}+2 \alpha_{21}^{2} \alpha_{22}^{2} S_{12}+2 \alpha_{21}^{2} \alpha_{23}^{2} S_{13}+ \\
+2 \alpha_{21}^{3} \alpha_{23} S_{15}+\alpha_{22}^{4} S_{22}+2 \alpha_{22}^{2} \alpha_{23}^{2} S_{23}+ \\
+2 \alpha_{21} \alpha_{22}^{2} \alpha_{23} S_{25}+\alpha_{23}^{4} S_{33}+2 \alpha_{21} \alpha_{23}^{3} S_{35}+ \\
+\alpha_{22}^{2} \alpha_{23}^{2} S_{44}+2 \alpha_{21} \alpha_{22}^{2} \alpha_{23} S_{46}+ \\
+\alpha_{21}^{2} \alpha_{23}^{2} S_{55}+\alpha_{21}^{2} \alpha_{22}^{2} S_{66}
\end{array}\right] ;
\end{gathered}
$$

where $\alpha_{i j}$ is the guide cosines.

The formation of auxeticity surfaces can be explained by considering in more detail the angular dependence of the Poisson coefficients $\mu(\varphi, \theta, \psi)$, where the Euler angles set the matrix of guide cosines $\alpha_{i j}$. Changing the Euler angles at certain steps $\Delta \varphi, \Delta \theta, \Delta \psi$ sets all possible crystal orientations in space. As already noted, the negative Poisson coefficient values $(\mu<0)$ are concentrated, as a rule, around some directions and form in space the pointing auxeticity surfaces. The ratio of the number of crystal orientations at which $\mu<0$ to the total number of possible orientations determines the crystal auxeticity degree $S_{a}$.

\section{Results of studying auxetic properties}

Fig. 1-6 show the Young modulus characteristic surface $(a)$, the Poisson coefficient angular distribution $(b)$, and the pointing auxeticity surface $(c)$ of the examined single crystals in a monoclinic crystal system [9]. The values $\mathrm{E}(\mathrm{g}), \mu_{i j}$, and the pointing surfaces were calculated using ratios (2), (3) applying the experimental values $S_{i j}$, given in [16]. The arrangement of crystals in Fig. $1-6$ is shown in the order of ascending in their degree of auxeticity $S_{a}$.

The Young modulus characteristic surfaces in Fig. 1, $a-6, a$ form rather complex three-dimensional shapes that reflect the anisotropy of elastic properties and have all the elements of symmetry inherent in monoclinic single crystals.

The Young modulus extreme values are located along the axes $L_{2}$ or along the normal to the plane of symmetry $P$.

The numerical values of $E_{i}$ can be obtained by building the intersections of the characteristic surfaces with arbitrary planes $(h k l)$. The Poisson coefficient values can be determined in a random direction from ratio (3). However, it is impossible to build a four-dimensional Poisson coefficient $\mu(\varphi, \theta, \psi)$ characteristic surface in a three-dimensional coordinate system [9]. Given that the period of function $\mu(\varphi, \theta, \psi))$ for $\varphi$ is equal to $\pi$ and it is symmetrical relative to $\varphi=\pi / 2$, one can build the function $\mu(\varphi, \theta, \psi)$ at fixed values $\varphi=0, \pi / 2$ (Fig. $1, b-6, b$ ). This makes it possible to estimate both the minimum $\left(\mu_{\min }\right)$ and the maximum $\left(\mu_{\max }\right)$ values of the Poisson coefficients.

An analysis of ratios (2) and (3) shows that the highs of distribution curves $\mu(\varphi, \theta, \psi)$ are directed along the second-order axes $L_{2}$ and normals to the plane of single crystal symmetry. The minimum $\mu_{\min }$ and maximum $\mu_{\max }$ values of Poisson coefficients for the studied single crystals, determined from the distribution curves $\mu(\varphi, \theta, \psi)$, are given in Table 1.

Table 1

The degree of auxeticity $S_{a}$ and the extreme Poisson coefficient values

\begin{tabular}{|c|c|c|c|}
\hline Parameter Crystal & $S_{a}$ & $\mu_{\min }$ & $\mu_{\max }$ \\
\hline Labradorite & 0.005 & -0.09 & 0.66 \\
\hline $\mathrm{CaSo}_{4} 2 \mathrm{H}_{2} \mathrm{O}$ & 0.012 & -0.1 & 0.06 \\
\hline $\mathrm{ZrO}_{2}$ & 0.31 & -0.6 & 0.55 \\
\hline$\beta-\mathrm{F}_{6} \mathrm{H}_{4} \mathrm{~F}_{6}$ & 0.35 & -0.55 & 1 \\
\hline $\mathrm{CsH}_{2} \mathrm{PO}_{4}$ & 0.36 & -1.7 & 2.25 \\
\hline $\mathrm{LaNbO}_{4}$ & 0.43 & -3.0 & 4.0 \\
\hline
\end{tabular}

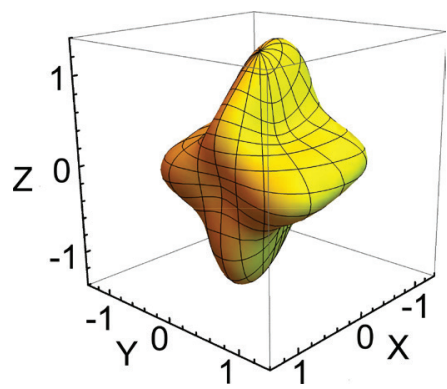

$a$

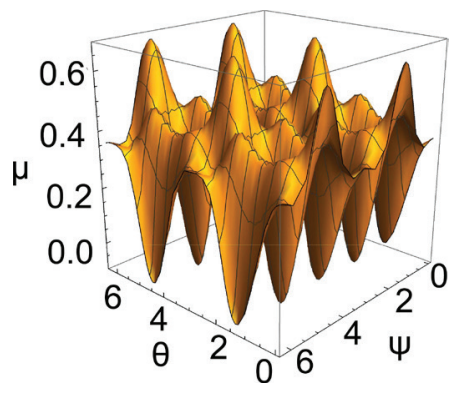

$b$

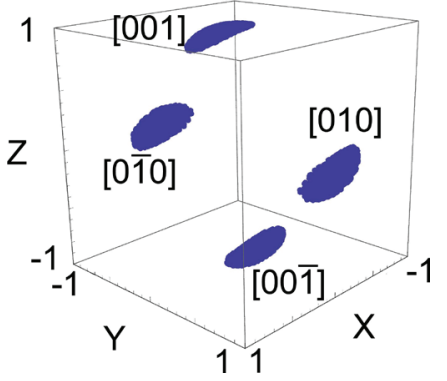

Fig. 1. Image for a labradorite single crystal $(\varphi=\pi / 2): a-$ the Young modulus characteristic surface; $b-$ the Poisson coefficient $\mu(\varphi, \theta, \psi)$ angular distribution; $c$ - pointing auxeticity surface 

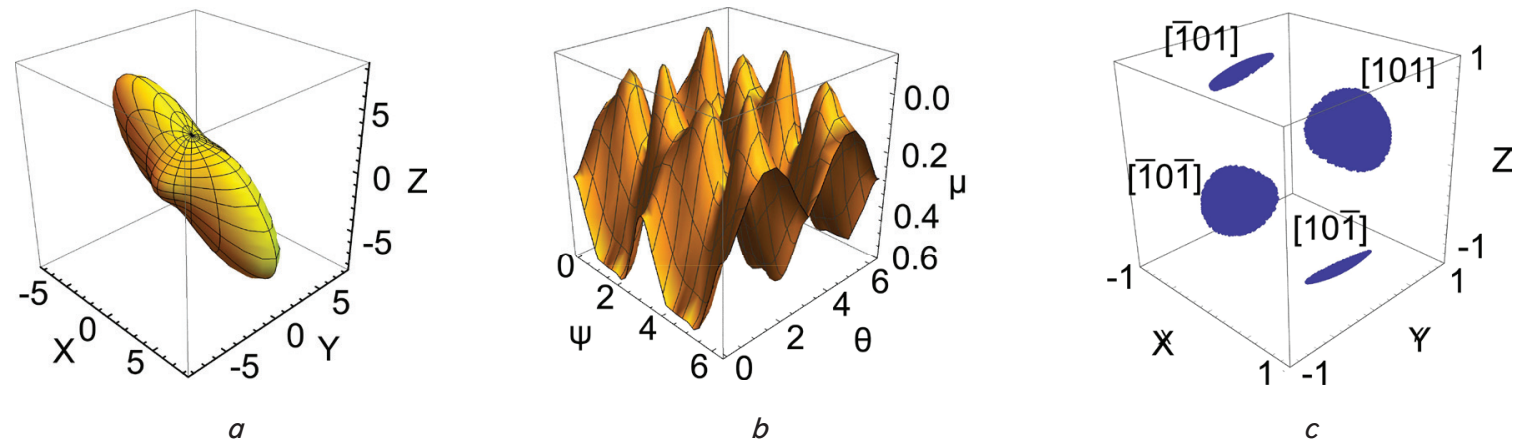

Fig. 2. Image of a calcium sulfate dihydrate single crystal along the normals to symmetry planes $P(\varphi=0): a-$ the Young modulus characteristic surface; $b-$ the Poisson coefficient $\mu(\varphi, \theta, \psi)$ angular distribution; $c-$ pointing auxeticity surface
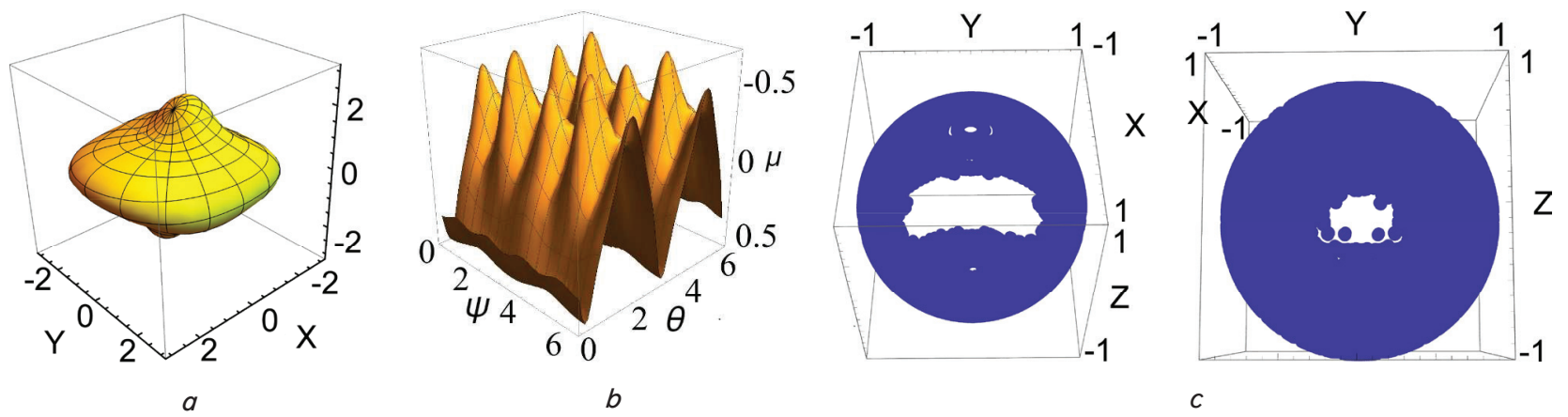

Fig. 3. Image of a $\mathrm{ZrO}_{2}$ single crystal $(\varphi=\pi / 2): a-$ the Young modulus characteristic surface; $b$ - the Poisson coefficient $\mu(\varphi, \theta, \psi)$ angular distribution; $c$ - pointing auxeticity surface
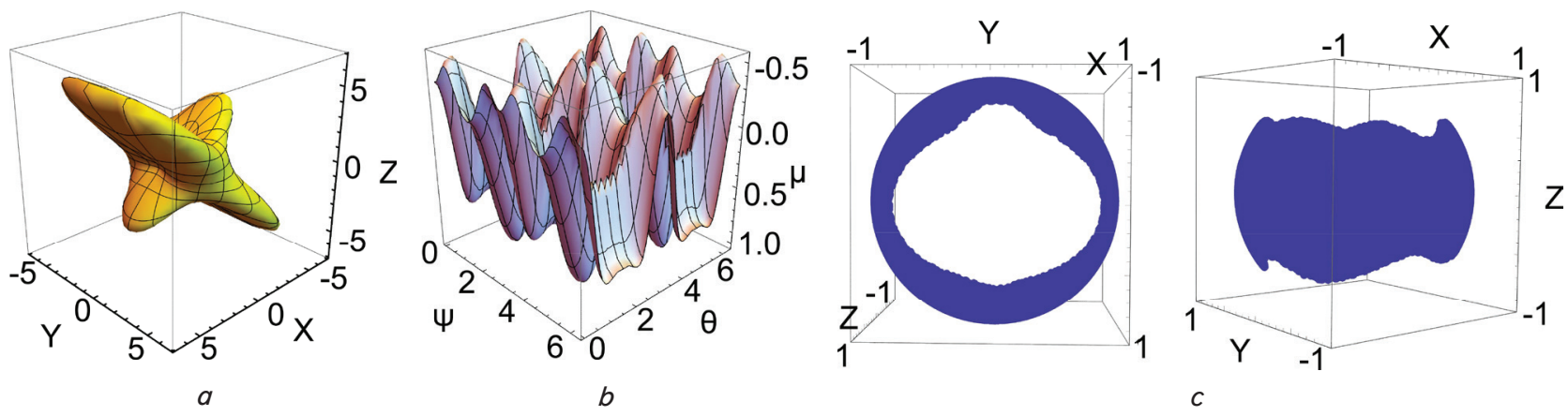

Fig. 4. Image of a $\beta-\mathrm{F}_{6} \mathrm{H}_{4} \mathrm{~F}_{6}$ single crystal $(\varphi=0): a-$ the Young modulus characteristic surface; $b-$ the Poisson coefficient $\mu(\varphi, \theta, \psi)$ angular distribution; $c$ - pointing auxeticity surfaces

An array of negative Poisson coefficient values forms in space the pointing auxeticity surfaces, which are shown in Fig. 1, $c-6, c$. For the single crystals of labradorite and sulfate dihydrate, there is a slight angular scattering of negative Poisson coefficient values while the pointing auxeticity surfaces are depicted in the form of individual spots in the [-101], [10-1], [-10-1] crystallographic directions for calcium sulfate dihydrate (non-axial auxeticity). Their degree of auxeticity $S_{a}$ is small -0.005 and 0.012 and, therefore, the minimum values of the Poisson coefficients $\left(\mu_{\min }\right)$ are equal to -0.09 and -0.1 , respectively. Positive values $\left(\mu_{\max }\right)$ reach the upper limit of acceptable values: $\mu_{\max }=0.66$ (for labradorite) and $\mu_{\max }=0.53$ (for Gypsum), (Table 1).

\section{Discussion of results of studying the auxetic properties of crystals in a monoclinic crystal system}

The negative Poisson coefficient values for $\mathrm{ZrO}_{2}$, $\beta-\mathrm{F}_{6} \mathrm{C}_{4} \mathrm{~F}_{6}$ single crystals are concentrated in 8 crystallo- graphic directions: axial $-<h 00>,<101>$ and non-axial $-<h k 0>,(h=k=+/-1)$ for each crystal. The pointing auxeticity surfaces of these single crystals is formed in the form of spherical segments. In Fig. 1, $c, 4, c$, it is shown in two projections. The degree of auxeticity $S_{a}$ increases significantly, accompanied by a rapid decrease in $\mu$ (from -0.1 to -0.6 ). The maximum value of the Poisson coefficients for a $\beta-\mathrm{F}_{6} \mathrm{C}_{4} \mathrm{~F}_{6}$ single crystal increases almost twice.

The negative Poisson coefficient values of single crystals in cesium dihydrogen phosphate $\mathrm{CsH}_{2} \mathrm{PO}_{4}$ are directed in 4 axial [010], [001], [0-10], and [00-1] and 4 non-axial [011], $[0-11],[0-1-1]$, and [01-1] directions, that is, there is a mixed auxeticity.

A similar situation occurs for the single crystals of lanthanum niobate: $\mu_{\min }<0$ are directed in 4 axial [010], [100],

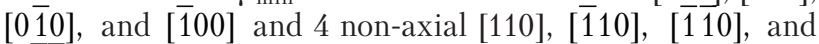
[110] directions. As a result of the imposition of the negative values of the Poisson coefficients, a rather complex pointing auxeticity surfaces are formed; for its detailed description, it is necessary to consider several projections (in Fig. 5, $c, 6, c$ ). 

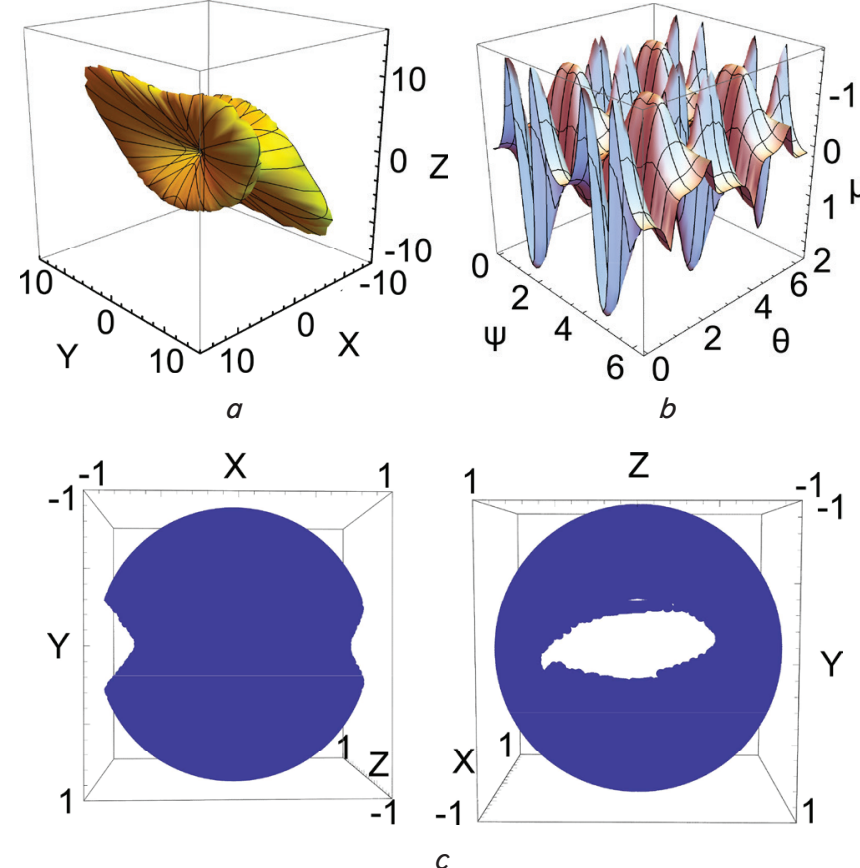

Fig. 5. Image of a $\mathrm{CsH}_{2} \mathrm{PO}_{4}$ single crystal $(\varphi=\pi / 2)$ :

$a$ - the Young modulus characteristic surface; $b-$ the angular distribution of Poisson coefficients $\mu(\varphi, \theta, \psi) ; c$ - pointing auxeticity surfaces
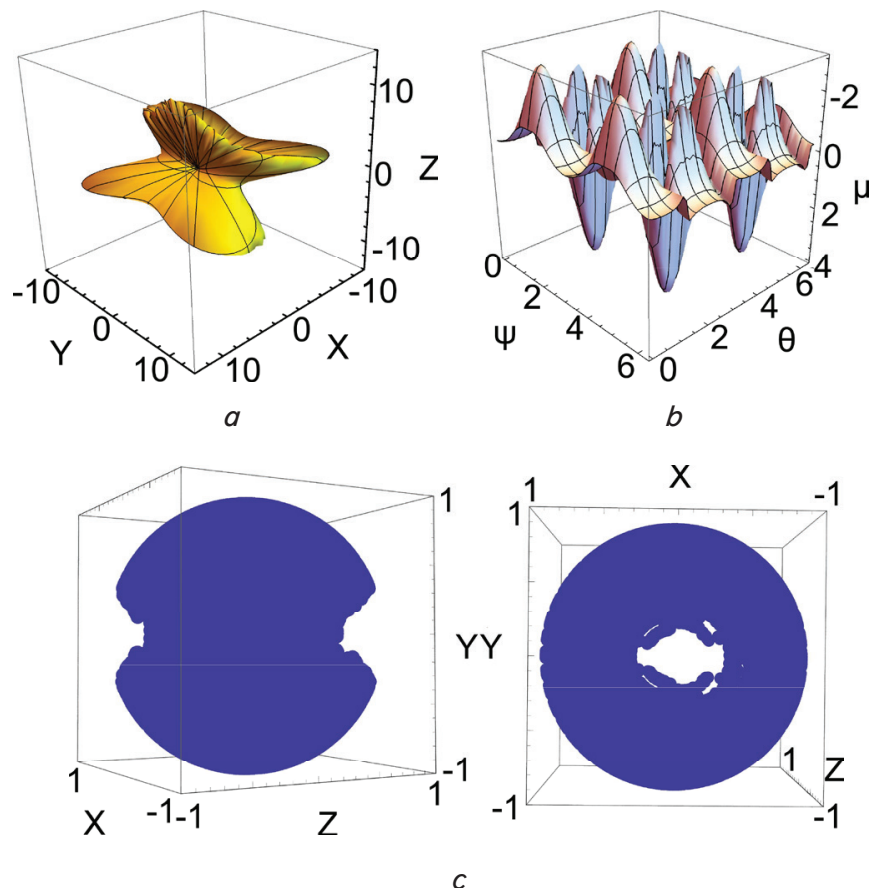

Fig. 6. Image of a $\mathrm{LaNbO}_{4}$ single crystal $(\varphi=\pi / 2): a-$ the Young modulus characteristic surface; $b$ - the angular distribution of Poisson coefficients $\mu(\varphi, \theta, \psi) ; c-$ pointing auxeticity surfaces
This also applies to the Young modulus characteristic surfaces. Along with the images of regions of the negative Poisson coefficient values, there are sufficiently long light fields where $\mu(\varphi, \theta, \psi)>0$. Consequently, these crystals are not absolute auxetics and their auxeticity degree $S_{a}$ is equal to 0.36 (for $\mathrm{CsH}_{2} \mathrm{PO}_{4}$ ) and 0.43 (for $\mathrm{LaNbO}_{4}$ ).

Note that with an increase in the degree of auxeticity $S_{a}$ there is a simultaneous decrease in the minimum $\mu_{\min }$ and an increase in the maximum $\mu_{\max }$ values of the Poisson coefficients (Table 1). For the $\mathrm{CsH}_{2} \mathrm{PO}_{4}$ and $\mathrm{LaNbO}_{4}$ single crystals, the $\mu_{\max }$ values exceed the maximum permissible boundary for isotropic crystals by 4 and 8 times, respectively. The second boundary is also exceeded by almost twice $-\mu_{\min }=-1.7\left(\mathrm{CsH}_{2} \mathrm{PO}_{4}\right)$ and three times $\mu_{\min }=-3.0\left(\mathrm{LaNbO}_{4}\right)$. Such abnormal values of the Poisson coefficients are due to the extremely high anisotropy of single crystals in a monoclinic crystal system. It should be noted that the extreme values of the Poisson coefficients for the single crystal of $\mathrm{LaNbO}_{4}\left(\mu_{\min }=-3.0 ; \mu_{\max }=4.0\right)$ are a record for crystals in all crystal systems [9].

In conclusion, we note that the mechanisms of abnormal deformations of single crystals of the low category are practically not studied. Low symmetry leads to the emergence of a large number of elastic coefficients and an increase in the auxetic directions at which $\mu_{\min }<0$. At the same time, most crystals are partial auxetics while the absolute auxeticity is quite rare and is mostly near the phase transition points.

\section{Conclusions}

1. We have built the pointing auxeticity surfaces of single crystals in the monoclinic crystal system of gypsum labradorite, zirconium oxide, fluoroduodecane, cesium dihydrogen phosphate, and lanthanum niobate. The experimental values of the Poisson coefficients are found to far exceed the minimum and maximum permissible boundaries for isotropic crystals $(-1 \leq \mu \leq 0.5)$.

2 . The mechanisms and patterns in the formation of the pointing auxeticity surfaces have been established. For partial auxetics, the negative Poisson coefficient values are concentrated only in certain crystallographic directions in the form of individual points or spots (Fig. 1,c). With the growth in the number of directions with negative values $\mu_{\min }<0$, the array of the negative Poisson coefficient values forms in space the auxeticity surface almost in the form of a sphere (Fig. 5, $c, 6, c$ ).

3. It is shown that the increase in the degree of auxeticity $S_{a}$ is accompanied by a simultaneous reduction in the minimum $\mu_{\min }$ and growth in the maximum $\mu_{\max }$ values of the Poisson coefficients.

4. It was found that the record extreme values of the Poisson coefficients are a consequence of the extremely high anisotropy of elastic properties of single crystals in a monoclinic crystal system.

1. Konek, D. A., Voytsehovski, K. V., Pleskachevskiy, Yu. M., Shil'ko, S. V. (2004). Materialy s otritsatel'nym koeffitsientom Puassona (obzor). Mehanika kompozitnyh materialov i konstruktsiy, 10 (1), 35-69.

2. Turley, J., Sines, G. (1971). The anisotropy of Young's modulus, shear modulus and Poisson's ratio in cubic materials. Journal of Physics D: Applied Physics, 4 (2), 264-271. doi: https://doi.org/10.1088/0022-3727/4/2/312

3. Goldstein, R. V., Gorodtsov, V. A., Lisovenko, D. S. (2010). Auxetic mechanics of crystalline materials. Mechanics of Solids, 45 (4), 529-545. doi: https://doi.org/10.3103/s0025654410040047 
4. Goldstein, R. V., Gorodtsov, V. A., Lisovenko, D. S. (2011). Young's modulus of cubic auxetics. Letters on Materials, 1 (3), $127-132$. doi: https://doi.org/10.22226/2410-3535-2011-3-127-132

5. Belomestnykh, V. N., Soboleva, E. G. (2011). Lateral strain ratios for cubic ionic crystals. Letters on Materials, 1 (2), $84-87$. doi: https://doi.org/10.22226/2410-3535-2011-2-84-87

6. Raranskyi, M. D., Balaziuk, V. N., Kovaliuk, Z. D. (2012). Pruzhni vlastyvosti ta dynamika krystalichnoi gratky deiakykh napivprovidnykovykh monokrystaliv. Chernivtsi: Zoloti lytavry, 200.

7. Raransky, M. D., Balazyuk, V. N., Melnyk, M. I., Gunko, M. M., Verebchan, Ya. S. (2014). The Peculiarities of Young's Modulus Surfaces of Cubic Single Crystals Formation. Physics and chemistry of solid state, 15 (4), 721-727.

8. Harrison, U. (1983). Elektronnaya struktura i svoystva tverdyh tel. Moscow: Mir, 381.

9. Raranskyi, M., Balaziuk, V., Hunko, M. (2016). Yavyshche auksetychnosti v tverdykh tilakh. Chernivtsi: «Druk Art», 178.

10. Lisovenko, D. S., Gorodtsov, V. A. (2011). Cubic crystals with negative Poisson’s ratio (cubic auxetics). Vestnik Nizhegorodskogo universiteta im. N. I. Lobachevskogo, 4 (2), 488-489.

11. Goldstein, R. V., Gorodtsov, V. A., Lisovenko, D. S. (2014). Young's modulus and Poisson's ratio for seven-constant tetragonal crystals and their nano/microtubes. Fizicheskaya mezomehanika, 17 (5), 5-14. doi: http://doi.org/10.24411/1683-805X-2014-00015

12. Raransky, M. D., Balazyuk, V. N., Gunko, M. M. (2016). Criteria and Mechanisms of Appearance of Auxeticity in Cubic Syngony Crystals. Metallofizika i noveishie tekhnologii, 37 (3), 379-396. doi: https://doi.org/10.15407/mfint.37.03.0379

13. Raransky, M. D., Balazyuk, V. N., Gunko, M. M. (2015). Auxeticity Properties of Hexagonal Syngony Crystals. Physics and chemistry of solidstate, 16 (1), 34-43. doi: https://doi.org/10.15330/pcss.16.1.34-43

14. Raransky, M. D., Tretiak, C. R., Gunko, M. M., Balazyuk, V. N. (2016). The Impact of d- and f-compression on Anisotropy of Elastic Properties of Single Crystals with Hexagonal Close Packing of Lattice. Physics and chemistry of solidstate, 17 (2), 170-179. doi: https://doi.org/10.15330/pcss.17.2.170-179

15. Raransky, M. D., Balazuyk, V. N., Gunko, M. M. (2015). Abnormal deformation properties of some single crystals of tetragonal syngony. Uzhhorod University Scientific Herald. Series Physics, 37, 8-19. doi: https://doi.org/10.24144/2415-8038.2015.37.8-19

16. Landolt-Börnstein. Numerical Data and Functional Relationships in Science and Technology. Group III: Crystal and Solid state Physics. Second and Higher Order Constants (1992). Berlin: Springer, 682.

17. Sirotin, Yu. I., Shaskol'skaya, M. P. (1975). Osnovy kristallofiziki. Moscow: Nauka, 680.

18. Ledd, M., Palmer, R. (Eds.) (1983). Pryamye metody v rentgenovskoy kristallografii. Moscow: Mir, 416

19. Landau, L. D., Lifshits, E. M. (1968). Teoriya uprugosti. Moscow: Nauka, 209.

20. Hearmon, R. F. S. (1956). The elastic constants of anisotropic materials - II. Advances in Physics, 5 (19), 323-382. doi: https:// doi.org/10.1080/00018732.1956.tadp0323

21. Kitaygorodskiy, A. I. (1971). Molekulyarnye kristally. Moscow: Nauka, 424. 